\title{
Comparison of BFS and Prim's Algorithm when used in MANETs Routing
}

\author{
Maria Jose \\ Department of Computer Science and Engineering \\ Mangalam College of Engineering \\ Kottayam, India
}

\author{
Subha Sreekumar \\ Department of Computer Science and Engineering \\ Mangalam College of Engineering \\ Kottayam, India
}

\begin{abstract}
One of the greatest challenge in MANETs for data transmission is route update. The route update in MANETs is implemented with the help of Breadth first search algorithm and Prim's algorithm. Using Prim's algorithm in route update mechanism will reduce the end-to-end delay to a greater extend in MANETs. The end-to-end delay can lead to a performance degradation in the network. Different algorithms can be used to update the route information in MANETs. The traditional approaches such as Breadth First Search algorithm used will increase the end-to-end delay since this algorithm will go through all the parent nodes before it goes to the children nodes.
\end{abstract}

Keywords: Prim's algorithm, MST, BFS, Route update.

\section{INTRODUCTION}

A MANET is a type of ad hoc network that can change locations. MANET network is not centralized, where all network activity including discovering the topology and delivering messages should be executed by the nodes themselves.

Mobile ad hoc networks (MANETs) can be called as a distributed network over a wide range of area and helps in the communication between people or devices without any preexisting infrastructure for communication [2]. Since there are chances of losing the route details the system should periodically send hello messages for observing the same. Once it is detected that the link to any node is lost the route should be reconstructed for the flawless transmission of data. Prim's algorithm provide a delay free mechanism for the route update. The system will first check for all the available nodes and will calculate the shortest path available using the Prim's algorithm. The shortest path obtained can be called as the minimum spanning tree (MST).

\section{RELATED WORKS}

MANETs can be used in different areas such as in traffic called Vehicular ad hoc networks (VANETs). VANETs are designed to provide safety to vehicles, monitor the traffic and many other applications. Due to the high mobility of vehicles there are chances of link failure frequently. Route update needs to be implemented frequently and fast during this time [3].

The packet routing in MANETs can be using proactive or reactive protocols. In proactive routing the nodes in the network will maintain valid routes to all destinations at all times where as in reactive a link to the destination is updated only when a node receives data from the upper layer [5].

The main examples for proactive routing protocols are OLSR and DSDV. Destination Sequenced Distance Vector (DSDV) transmits the data packets using the routing tables [7]. This table driven protocol is built based on the Bellman-Ford algorithm. The routing information is distributed among nodes using differential messages. The full dump messages are sent less frequently than the full dump messages.
Optimized link state routing (OLSR) usually minimizes the overhead of flooding by using multi point relays (MPRs). MPRs are nodes selected by neighbor nodes which will control the traffic of flooding by reducing the number of transmissions required. The link state information is provided by MPR to all nodes [6].

MANETs uses opportunistic data forwarding which refers to the way in which data packets are handled in a multi hop wireless network. A transmitter picks the best forwarder from multiple receivers and explicitly requests the selected node to forward data [4].

\section{ROUTE UPDATE}

To transmit the data packets from source to destination every nodes including the intermediate nodes should have an idea regarding the path to be followed. To accomplish this all nodes will broadcast their route information to all other nodes. This will lead to congestion in the network which can be avoided by carrying out differential updates. The route update messages are send by the nodes only when some significant updates occur [1].

For the route update Prim's algorithm and also BFS algorithm is also implemented. When the path is updated using BFS it could be seen that the end-to-end delay is higher than when the path is updated using the Prim's algorithm.

\subsection{Prim's Algorithm}

Prim's algorithm is a greedy algorithm which computes the (minimum spanning tree) MST of any connected edgeweighted graph. An edge-weighted graph is a graph where weights are associated with each edge. The MST constructed using Prim's algorithm is a spanning tree whose weight will be the least compared to all other spanning trees.

The minimum spanning tree will be connected and acyclic. Adding an extra edge to the MST will create a cycle for sure thus proving the MST is maximally acyclic. Also removing an edge from the MST will break the spanning tree into separate trees. Such group of edges which will separate the MST into sub trees are called cut of a connected graph. This property will make the MST minimally connected.

While creating an MST the algorithm can choose a random vertex initially and keeps on adding the vertices until the MST 
is created. The added vertex should be a new vertex which was not there in the constructed tree. This will add a new edge which connects the new vertex with a vertex which was already there in the constructed tree. Such an edge is called a crossing edge. The set of edges which are not included in the constructed MST are called ineligible edges.

\subsubsection{Implementation of Prim's Algorithm}

Prim's algorithm starts with an empty spanning tree. It maintains two sets of vertices, first set contains the vertices already included in the MST and the second contains the vertices not yet included in the MST. Let us define the graph as $G(V, E)$. $U$ is the non-empty subset of the vertex set $V$ which are already included in the MST and U-V is the set of vertex which are not part of the MST. Once the minimum weight edge is found out from the vertex set $(\mathrm{U}, \mathrm{V}-\mathrm{U})$ the new vertex if not part of the non-empty set $U$ is added to the same. The process is repeated until all the vertex has been included in the MST. The process has been shown in Figure 1

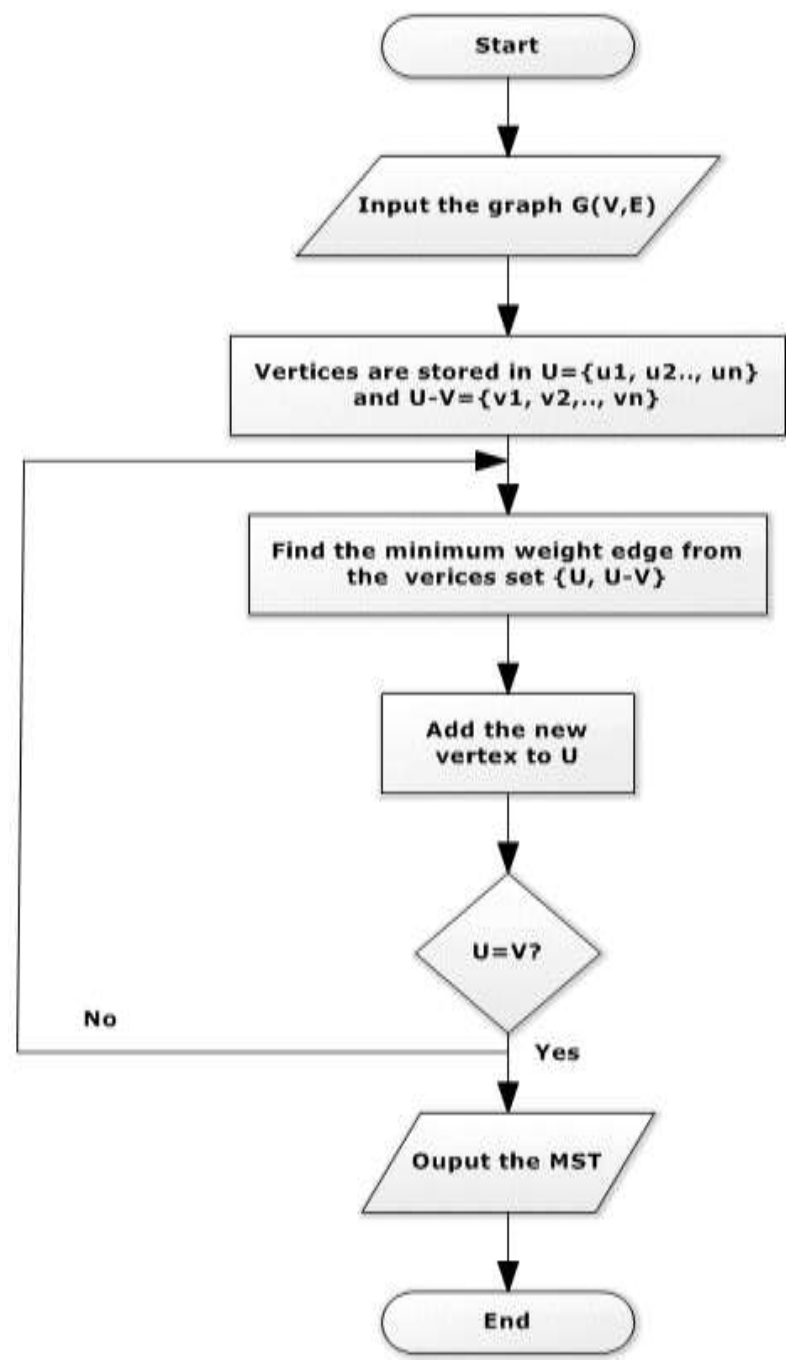

Figure 1. Flow chart of Prim's Algorithm

\subsubsection{Example for constructing MST using Prim's Algorithm}

Figure 2 will show an example of how to construct an MST using Prim's algorithm. At first a random node A is selected and all the edges emerging from $\mathrm{A}$ is marked. Then the least weighted edge is added to the tree. The minimum weighted edge is $\mathrm{AC}$ with weight 2 . Hence node $\mathrm{C}$ is added to the tree. Now all the edges from $\mathrm{C}$ is marked. Again the process of finding the minimum weighted edge from all the marked edges is found out and the corresponding node F is added. At any time if we encounter edge of same weight an arbitrary edge can be selected. The process will continue until all the nodes are added to the tree. Finally MST with all nodes are constructed.

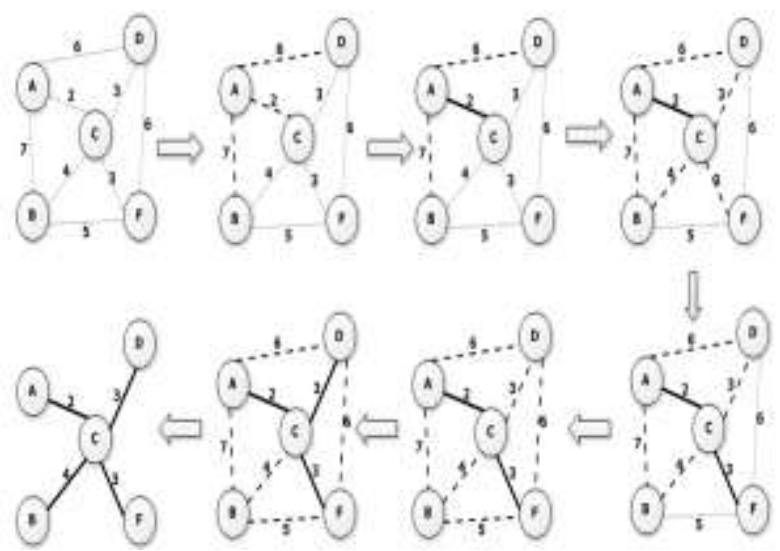

Figure 2. Example for constructing MST

\subsection{Breadth First Search Algorithm}

Breadth First Search (BFS) is an easy algorithm. BFS will build a tree by adding all children of the starting vertex before it will add the grandchildren. Hence the BFS traversal in a graph will produce a breadth first search tree. The main aim of BFS is to reach the root node as soon as possible by traversing the graph.

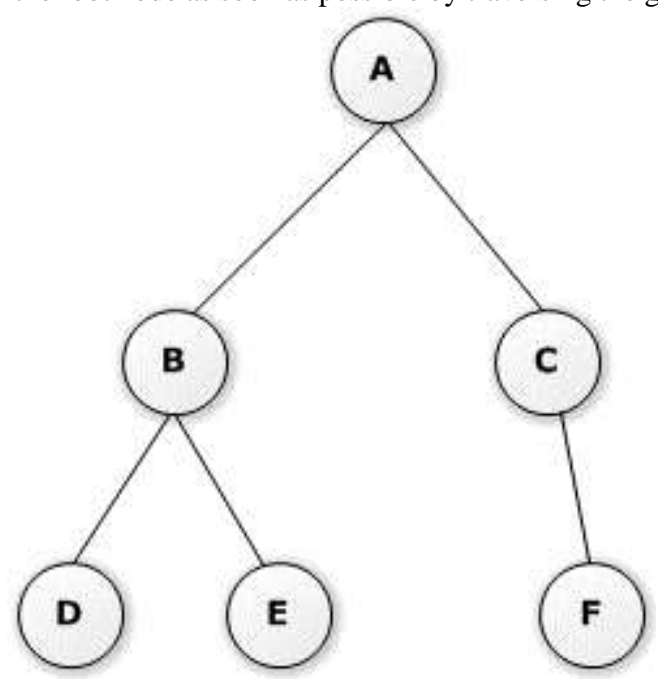

Figure 3. Breadth First Search Tree

If a traversal is done through the graph given in figure 3 the output will be printed as A B C D E F. That is the traversal in BFS is by levels. The BFS will visit the nodes at higher level before visiting the nodes at lower level.

\section{EXPERIMENTAL RESULTS}

Experiments were carried out to compare the route update using breadth first search and Prim's algorithm. The simulation network was developed in java and random values are assigned for each node. The development platform used was Net Beans 
and the database used was MySQL. Experiments are conducted on Intel Core i5 processor and 12GB of RAM in Windows 7 OS.

At first route update has been done with the help of breadth first search algorithm in the existing nodes. Later for the same nodes route update is done with Prim's algorithm and calculated the minimum spanning tree. The evaluation result obtained in Figure 4 shows that the end-to-end delay in route update using Prim's algorithm is comparatively very less compared to the route update done using breadth first search.

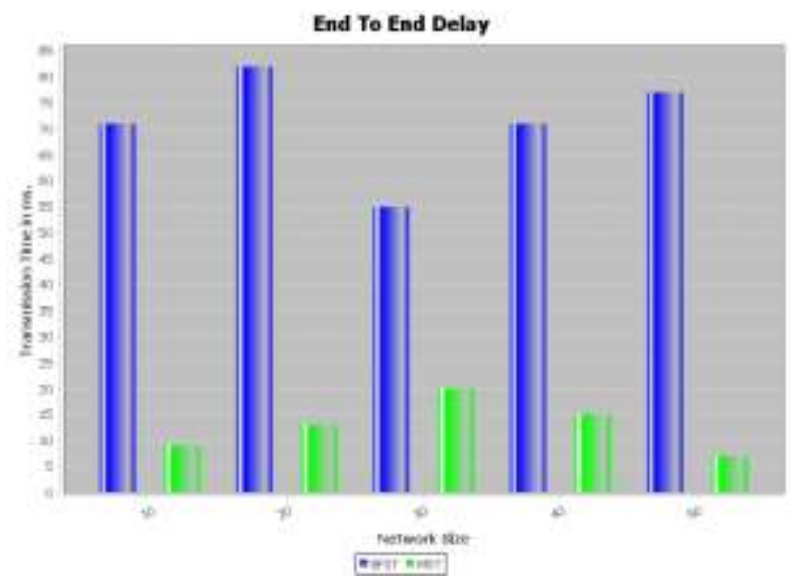

Figure 4. BFS vs. Prim's Algorithm

\section{CONCLUSION AND FUTURE SCOPE}

Whenever some request comes for packet delivery the preexisting route is selected for data flow. The path information needs to be updated in MANETs whenever some failure occurs. The route updated using Prim's algorithm will be a minimum spanning tree which will reduce the end-to-end delay there by improving the performance of the system compared to BFS.

The data packets are retransmitted whenever some failure occurs which will contributes to the congestion of network. Instead of retransmitting, the data packets can be recovered using some local mechanisms in the near future.

\section{REFERENCES}

[1] Zehua Wang, Yuanzhu Chen, and Cheng Li, "PSR: A light weight Proactive Source Routing Protocol for Mobile Adhoc Networks, IEEE 2014

[2] I. Chlamtac, M. Conti, and J.-N. Liu, "Mobile ad hoc networking: Imperatives and challenges," Ad Hoc Netw., vol. 1, no. 1, pp. 13-64, Jul. 2003.

[3] M. Al-Rabayah and R. Malaney, "A new scalable hybrid routing protocol for VANETs," IEEE Trans. Veh. Technol., vol. 61, no. 6, pp. 2625-2635, Jul. 2012.

[4] Y. P. Chen, J. Zhang, and I. Marsic, "Link-layer-andabove diversity in multi-hop wireless networks," IEEE Commun. Mag., vol. 47, no. 2, pp. 118-124, Feb. 2009.

[5] S. Biswas and R. Morris, "ExOR: Opportunistic multi-hop routing for wireless networks," in Proc. ACM Conf. SIGCOMM, Philadelphia, PA, USA, Aug. 2005, pp. 133144.

[6] T. Clausen and P. Jacquet, "Optimized Link State Routing Protocol (OLSR)," RFC 3626, Oct. 2003.
[7] C. E. Perkins and P. Bhagwat, "Highly dynamic Destination-Sequenced Distance-Vector Routing (DSDV) for mobile computers," Comput. Commun.Rev., vol. 24, pp. 234-244, Oct. 1994. 